NP25 (continued)

to promote healthy eating can support healthy BMI growth trajectories in children.

Funding: 2015-68001-23232

\section{NP26 Farm Fresh Foods for Healthy Kids: Innovative Cost-Offset Community Supported Agriculture Intervention to Prevent Childhood Obesity and Strengthen Local Agricultural Economies}

Rebecca A. Seguin, PhD, CSCS, rs946@cornell.edu, Cornell University, 412 Savage Hall, Cornell University, Ithaca, NY 14853; Alice S. Ammerman, DPH, University of North Carolina at Chapel Hill; Karla L. Hanson, PhD, Cornell University; Jane Kolodinsky, PhD, University of Vermont; Stephanie B. Jilcott Pitts, PhD, East Carolina University; Marilyn Sitaker, MPH, The Evergreen State College

Objective: To test the impact of cost-offset community supported agriculture (CO-CSA) on availability, accessibility, preparation skills, and consumption of fruits and vegetables (FV) as a pathway to childhood obesity prevention. Description: This community-based CO-CSA intervention includes: 15-24 week summer produce share at 50\% price reduction; weekly SNAP/EBT or cash balance payments of \$8-\$21 depending upon size selected; choice of 2-4 large kitchen tools; and nine CSA-tailored education classes. Households were eligible if: income $<185 \%$ of the federal poverty level, had not participated in a CSA for $>3 y$ (or ever), included a child $2-12 y$ who also was willing to participate, and paid a deposit equivalent to two weekly balance payments.

Evaluation: Two-hundred households (53\% of eligible) enrolled in spring 2016 and were randomly assigned to intervention (CO-CSA in 2016 and 2017) or delayed intervention (CO-CSA in 2017 and 2018). Groups were comparable in size and characteristics (all $\mathrm{P}>0.05$ ). Most adults were women $(97 \%), 31-50 y(68 \%)$, and half graduated from college (49\%). Adults scored between neutral and confident in eating and cooking FV; some reported difficulty financially affording FV (30\%). At baseline, median FV intake was 2.8c for adults and 2.9c for children. Most participants picked-up at least three-quarters of their weekly CSA shares (57\%), and attended at least one CSAtailored education class (68\%). Most households (78\%) provided follow-up data in fall 2016.

Conclusions and Implications: Additional recruitment into the intervention will conclude in summer 2017, total recruitment target of 240 households enrolled at 13 farms across 4 states. Preliminary findings will be available in 2018.

Funding: USDA-NIFA 2015-68001-23230

\section{NP27 Clearing the Path to the Prevention of Childhood Obesity: How to Expedite the Track \\ Margarita Teran-Garcia, PhD, MD, teranmd@illinois.edu, University of Illinois Urbana-Champaign, 216 Child}

Development Laboratory, Urbana, IL 61801; Angela Wiley, PhD, University of Illinois Urbana-Champaign; Bridget Hannon, BS; Elizabeth Villegas, BA, Abriendo Caminos Research Team

Objective: To adapt the Abriendo Caminos (AC2) curriculum for families of Hispanic-heritage to include aspects of social cognitive theory proven to be successful in similar interventions (SCT), current nutrition evidence-based research (NEBR), and cultural tailoring from structured focus group and community feedback (CTC).

Description: AC2 is a multistate, randomized controlintervention program developed to improve nutrition, physical activity, and family interactions. The intervention consisted of two-hour workshops for six weeks, Participants answered a survey before randomized group was assigned. Weight and height were measured by trained personnel.

Evaluation: We report findings from families recruited in Illinois $(n=53)$, and present changes in a healthy eating adherence index for the key recommendations of the 2015 Dietary Guidelines for Americans. The prevalence of overweight/obesity in participating children (22\% / 35\%) and adults (37\% / 55\%) surpasses the national averages. After six weeks, mothers participating in the intervention reported increased consumption low-fat dairy, compared to those in the control group $(\mathrm{p}<0.05)$. Examples of adaptations included: SCTrelated, dietary self-efficacy implemented through skill building activities, where participants were allowed to serve themselves and practice age-appropriate portion sizes; NEBR-related where replacement of saturated for unsaturated fat in the diet was demonstrated, and CTC-related where information about organic foods was added after participants expressed interest to better understand this topic using examples of traditional Hispanic foods.

Conclusions and Implications: Culturally tailored education programs and interventions are urgently needed to not only teach families ways to promote health, but also to increase mothers' efficacy to prevent and manage obesity in their children.

Funding: 2015-68001-23248

\section{NP28 Obesity Risk, Parenting and Diet Quality Tools for Hispanic Families with Preschoolers: EFNEP, Head Start, Medical Clinic-Year 2}

Marilyn Townsend,PhD, RD, mstownsend@ucdavis.edu, University of California, Davis, 1 Shields Avenue, Davis, CA 95616; Mical Kay Shilts, PhD, California State University, Sacramento; Lenna Ontai, PhD, University of California, Davis; Christana Drake, PhD;

Louise Lanoue, PhD; Dennis Styne, MD; Lindsay Allen, PhD, Western Human Nutrition Research Center; Karina Dias Rios, PhD, University of California, Merced; Katherine Panarella, MPH, MS, University of California Cooperative Extension Agricultural and Natural Resources 